\title{
Poloidal asymmetries in the edge density profiles on ASDEX-Upgrade
}

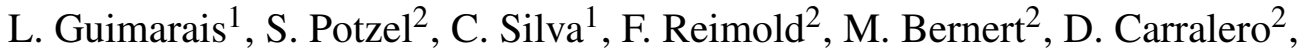

G. D. Conway ${ }^{2}$, M. E. Manso ${ }^{1}$, V. Nikolaeva ${ }^{1,3}$, J. Santos $^{1}$, A. Silva ${ }^{1}$, E. Wolfrum ${ }^{2}$, the EUROfusion MST1 Team* and the ASDEX-Upgrade Team

${ }^{1}$ Instituto de Plasmas e Fusão Nuclear, Instituto Superior Técnico, Universidade de Lisboa, Portugal, ${ }^{2}$ Max-Planck-Institut für Plasmaphysik, Boltzmannstr. 2, 85748, Garching, Germany, ${ }^{3}$ Technische Universität München, James-Franck-Str. 1, 85748, Garching, Germany, * See http://www.euro-fusionscipub.org/mst1

\section{Introduction}

Poloidal asymmetries in the midplane edge density profiles develop according to the divertor detachment state in the ASDEX-Upgrade Tokamak (AUG)[1]. The appearance of a High Field Side High Density Front (HFSHD) associated with inner divertor (ID) detachment has been reported [2,3]. This HFSHD is located in the Scrape-Off Layer (SOL), at the height of the X-point, and can propagate upwards to the midplane, as measured by the Low-Field-Side (LFS)/High-Field-Side (HFS) O-mode Reflectometer.

\section{Evolution of the divertor parameters and midplane edge density profiles}

The typical evolution of the plasma parameters measured at the divertor and SOL during a density ramp is shown in figure 1 for a L-mode discharge with lower single null, plasma current of $I_{p}=0.8 \mathrm{MA}$ and a toroidal field of $\left|B_{T}\right|=2.5 \mathrm{~T}$. As characterized in [2], it is possible to experimentally classify divertor detachment in at least three states: the onset state (OS), fluctuating state (FS) and complete detachment state (CDS), with higher Degrees of Detachment, respectively. The Degree of Detachment (DoD) is defined as the ratio of the calculated $\left(\Phi_{D+}^{\text {calc }}\right)$ and measured $\left(\Phi_{D+}^{\text {meas }}\right)$ ion flux to the target, where $\Phi_{D}^{\text {calc }}+$ is assumed to be proportional to the square of the line-integrated plasma density [2]. Typically around the transition to the FS the peak density in the inner divertor volume measured by Stark broadening increases, moves from the target towards and even above the X-point and a region of high density develops in the volume of the inner far SOL. At the transition to complete detachment the HFSHD at the strike-point region disappears and moves towards the wall above the Xpoint. It was shown in [1] that there is a good agreement between the formation of the HFSHD at the divertor and at the midplane. Until the flattop at $1.1 s$, there is no symmetry at the midplane due to the formation process of the plasma column. For Ohmic discharges, density profiles measured by reflectometry are roughly HFS/LFS symmetric during the flattop [1]. 


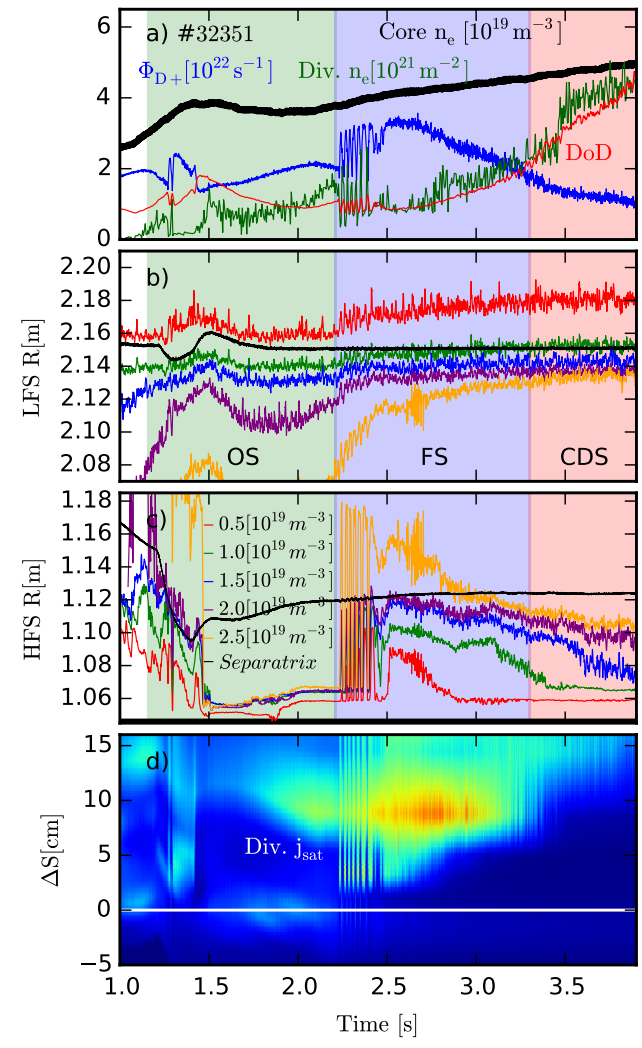

Figure 1: L-mode density ramp a) DoD, total $D$ flux in the $I D \Phi_{D^{+}}$, inner divertor density Div. $n_{e}$; b) and c): LFS and HFS iso-density layers from reflectometry. respectively; d) ID $j_{\text {sat }}$ distribution.

With $400 \mathrm{~kW}$ ECRH power applied from $t=0.9 \mathrm{~s}$, these discharges are never in such conditions. For $t>1.5 \mathrm{~s}$, still during the OS, the SOL density suddenly increases at the HFS leading to large poloidal asymmetries. Around $t=2.2 s$ the divertor plasma is observed to oscillate back and forth between the OS (early OS phase where the divertor is attached no HFSHD exists) and the FS. As illustrated in figure 1 these oscillations reach the HFS midplane leading to a strong modulation of the HFS density profiles. The evolution of the midplane edge density profile in the HFS is observed to respond to divertor oscillations, changing significantly for different divertor detachment states. The divertor HFSHD is seen to disappear periodically, coincident with the evolution of the midplane edge density profiles (see figure 1). In the OS a broad density front is observed across the divertor region extending to the farSOL while during the FS the density tends to decrease and move closer to the separatrix (disappearing at the far-SOL) in agreement with the evolution of the HFS upstream profiles (see figure $2 b$ ). As detachment progresses and the CDS is approached the far-SOL density increases again both at the divertor and midplane.

\section{Possible effect of inner wall recycling on the formation of the HFSHD}

Due to the proximity of the plasma at the midplane to the inner wall (IW), the role of recycling at the wall surface cannot be excluded. SOL density profiles broaden as the line-averaged density increases and may lead to a strong IW interaction. The possible contribution of an enhanced plasma-wall interaction to the formation of the midplane HFSHD was investigated by performing discharges with different IW clearances while keeping the strike-point location as constant as possible so that changes in divertor detachment are minimized. Two discharges, one with small wall clearance (32349: $4.5 \mathrm{~cm}$ inner clearance) and another with large (32351: $8.5 \mathrm{~cm}$ ) are presented in this work. To further classify detachment conditions, an asymmetry parameter, $\alpha$, obtained from density profiles only, is defined as schematically illustrated in figure 2a. It is the difference between the integrals of the HFS and LFS density profiles in the $\rho$ poloidal chord in the common radial range for each individual profile. 
Larger HFS than LFS densities will result in a positive contribution to the parameter (area in red), while the opposite will result in a negative contribution (small area in blue). To evaluate the effect of the inner wall clearance on the midplane HFSHD and on the detachment process parameters such as the DoD, asymmetry parameter and the inner divertor volume density are compared in figure 3 for the two discharges. The asymmetry is observed to increase significantly during the OS, to decrease during the FS $\left(n_{e} \approx\right.$ $3.5 \times 10^{19} \mathrm{~m}^{-3}$ ) where the divertor oscillations are observed, increasing again for $n_{e}>4 \times 10^{19} \mathrm{~m}^{-3}$ when the CDS is approached. The asymmetry parameter was not found to depend on the clearance for densities up to $4.5 \times 10^{19} \mathrm{~m}^{-3}$, suggesting

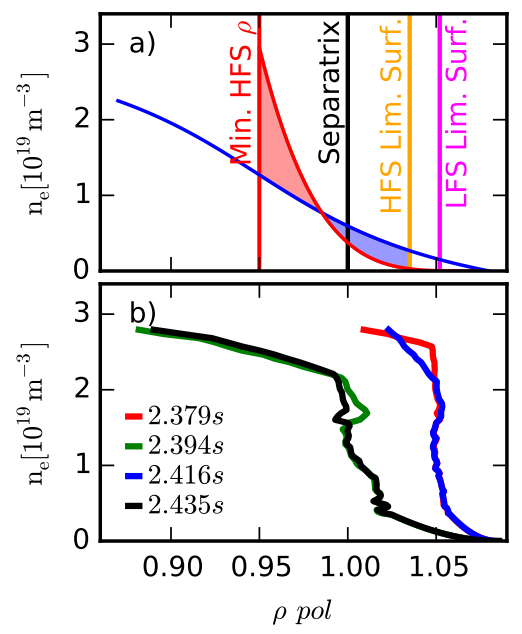

Figure 2: a): Simplified scheme for the definition of the asymmetry parameter; b) HFS density profiles during the two phases of the divertor oscillation. that the inner wall recycling does not play a significant role in the formation of the HFSHD.

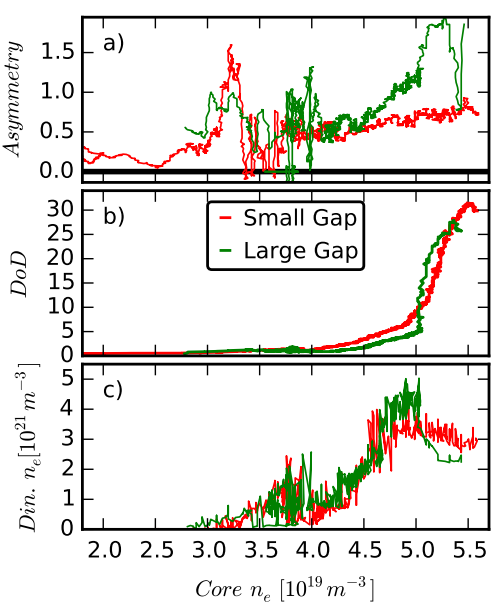

Figure 3: a): Asymmetry parameter $\alpha$; b) DoD; c) Inner divertor density.
However, at densities above $5 \times 10^{19} \mathrm{~m}^{-3}$, a larger asymmetry is observed for the higher clearance, possibly a result of boundary conditions (low distance to the wall may limit the density at the separatrix). It is found that the dominant role in the HFSHD formation is played by the inner divertor detachment rather than the plasma-wall interaction at the midplane. The inner gap clearance also appears to affect the HFSHD with slightly higher density observed for discharges with large clearance coincident with the evolution of the midplane edge density profiles. The reduction of the divetor volume density at the end of the discharge is justified by the density front moving up, outside the field of view of the diagnostic.

\section{Influence of the main Ion species and $\mathrm{N}_{2}$ seeding}

The influence of the main ion species in the development of the HFSHD was studied for Ohmic and L-mode density ramp discharges with Deuterium, Hydrogen and Helium. The asymmetry parameter at $n_{e}=4.5 \times 10^{19} \mathrm{~m}^{-3}$ was used to quantify the HFS/LFS density asymmetry (highest density common to all selected discharges). The dependence of the asymmetry on the plasma parameters studied is summarized in table 1. The same qualitative trend of the HFSHD front formation is observed in $\mathrm{H}$ and $\mathrm{D}$. The midplane asymmetries in the density profile have a weak dependence on the isotopic mass, only slightly higher for $\mathrm{H}$ plasmas, which agrees with 


\begin{tabular}{|c|c|c|c|}
\hline Shot & Characteristics & Asymmetry & Comparison \\
\hline$\overline{27360}$ & Ohmic Hydrogen & $\overline{0.3}$ & \multirow{2}{*}{$\mathrm{H} / \mathrm{D}$} \\
\hline 27098 & Ohmic Deuterium & 0.25 & \\
\hline 29865 & Deuterium, $P_{E C R H}=0.55 \mathrm{MW}$ & 1.0 & \multirow{2}{*}{$\mathrm{D} / \mathrm{He}$} \\
\hline 32792 & Helium, $P_{E C R H}=0.65 M W$ & 0.1 & \\
\hline 30801 & Deuterium with $N_{2}$ seeding, $P_{E C R H}=0.9 M W$ & 0.15 & \multirow{2}{*}{$N_{2}$ seeding } \\
\hline 31364 & Deuterium without $N_{2}$ seeding, $P_{E C R H}=0.9 \mathrm{MW}$ & 0.5 & \\
\hline
\end{tabular}

Table 1: Dependence of the asymmetry on the studied plasma parameters. The asymmetry parameter at $n_{e}=4.5 \times 10^{19} \mathrm{~m}^{-3}$ has been used to quantify the HFS/LFS density asymmetry.

larger densities in the divertor volume. A larger reduction in the midplane HFS/LFS asymmetry is found when comparing $\mathrm{D}$ with He plasmas in agreement with the observation that $\mathrm{He}$ plasmas exhibit the characteristics of detachment at a higher density. For the cases when Stark Broadening from Balmer lines (inner divertor density measurements) is unavailable, such as $\mathrm{He}$ plasmas, density in the divertor volume cannot be estimated. In AUG, it is also possible to puff impurities to cool down the divertor plasma via line radiation. It is observed that both the CDS occurs at a lower density ( $n \approx 3 \times 10^{19} \mathrm{~m}^{-3}$ ) while the poloidal asymmetry in the midplane density profile is modest. In addition, no indication of the divertor HFSHD is also observed (table 1). Given that the occurrence of the HFSHD seems to require a minimum level of additional heating power, the injection of $N_{2}$ would lead to the cooling of the divertor and consequently to the reduction of the HFSHD. Using the asymmetry parameter it is possible to corroborate previous findings [3], albeit far from the divertor.

\section{Summary}

The inner divertor is found to be the main player in the formation of the HFSHD at the midplane. Whilst the role of IW recycling cannot be completely discarded, its effect does not display a clear influence on experimental results. By defining an asymmetry parameter based solely on reflectometry data, the possibility to expand the study to the isotopic effect on the formation of the HFSHD was demonstrated, revealing a smaller HFSHD for He plasmas.

\section{Acknowledgements}

This work has been carried out within the framework of the EUROfusion Consortium and has received funding from the Euratom research and training programme 2014-2018 under grant agreement No 633053. The views and opinions expressed herein do not necessarily reflect those of the European Commission. IPFN activities also received financial support from "Fundação para a Ciência e Tecnologia" through project UID/FIS/50010/2013 and grant SFRH/BD/87738/2012.

\section{References}

[1] L. Guimarais et. al., EPS 2015

[3] S. Potzel et al, Journal of Nuclear Materials (2015)

[2] S. Potzel et al, Nuclear Fusion 54 (2014) 013001 